Produto \& Produção, vol. 16 n.1, p. 48-65, mar. 2015

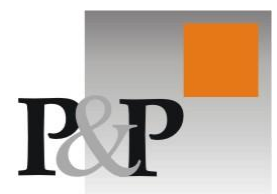

SUBMETIDO EM 17/01/2014. ACEITO EM 30/03/2015.

\title{
Níveis de conhecimento no processo decisório: uma avaliação a partir do método CWS
}

\author{
Mauri Leodir Löbler \\ Universidade Federal de Santa Maria - UFSM \\ lobler@ccsh.ufsm.br
}

Eliete dos Reis Lehnhart

Universidade Federal de Santa Maria - UFSM

elietedosreis@gmail.com

Taís de Andrade

Universidade Federal de Santa Maria - UFSM

tais0206@gmail.com

Vânia de Fátima Barros Estivalete

Universidade Federal de Santa Maria - UFSM

vaniafbe@terra.com.br

\section{RESUMO}

Os níveis de conhecimento e especialização dos indivíduos envolvidos em processos decisórios assumem grande relevância em diversas áreas do contexto organizacional. Há uma preocupação na literatura sobre a mensuração do nível de conhecimento especializado, sendo apontadas limitações no uso dos métodos tradicionais para mensurar tais níveis (WEISS et al., 2006; WITTEMANN et al., 2012). Diante deste contexto, o objetivo deste estudo é avaliar a consistência e a discriminação de indivíduos com diferentes níveis de conhecimento no processo decisório, considerando a complexidade da tarefa. Assim, por meio de uma pesquisa experimental, foram aplicadas duas tarefas decisórias (tarefa simples e tarefa complexa), relacionadas à simulação de compra de um imóvel residencial, a indivíduos especialistas e não especialistas. O cálculo do índice CWS, o qual mensura o nível de conhecimento especializado, evidenciou que os não especialistas discriminaram mais e foram menos inconsistentes, apresentando valores superiores do índice CWS.

Palavras-chave: níveis de conhecimento; processo decisório; índice CWS (Cochran-Weiss-Shanteau).

\begin{abstract}
The levels of knowledge and expertise of the individuals involved in decision-making processes are of great importance in many areas of organizational context. There is concern in the literature on measuring the level of expertise knowledge, being identified out limitations in the use of traditional methods to measure such levels (WEISS et al., 2006; WITTEMANN et al., 2012). Given this context, this study aims to evaluate the consistency and discrimination of individuals with different levels of knowledge in decision making, considering the complexity of the task. Thus, through an experimental
\end{abstract}


study were applied two decision-making tasks (simple task and complex task), related to the simulation of purchasing a residential property to individuals specialists and non-specialists. The calculation of the CWS index, which measures the level of knowledge skilled, showed that the nonexperts discriminated more and were less inconsistent, exhibiting higher values of the CWS index.

Keywords: levels of knowledge, decision making; CWS (Cochran-Weiss-Shanteau) Index

\section{Introdução}

Os níveis de conhecimento e especialização dos indivíduos envolvidos em processos decisórios assumem grande relevância nas diversas áreas do contexto organizacional. Conforme Malhotra et al. (2007), a qualidade da decisão está diretamente relacionada com o nível de conhecimento dos decisores sobre o assunto, sugerindo a importância da identificação e distinção correta de especialistas (LÖBLER e HOPPEN, 2008).

$\mathrm{Na}$ literatura associada ao processo decisório, percebe-se uma preocupação com a identificação de tais níveis, especificamente na diferenciação de especialistas e novatos, com a intenção de ampliar o entendimento acerca das diferenças cognitivas desses indivíduos (ERICSSON, 2006; CHEN et al., 2006; GERMAIN, 2011). Sob esta perspectiva, Chen et al. (2006) defendem que especialistas e novatos possuem diferentes características de aprendizagem e modelos mentais distintos, que exercem influência sobre suas escolhas e análise de informações relacionadas ao processo decisório.

Constata-se também que diferenças entre especialistas e novatos (neste estudo denominados de não especialistas) são relevantes para o entendimento de como o indivíduo se comporta em diferentes contextos relacionados à tomada de decisão. Conforme Löbler e Hoppen (2008), variáveis que afetam o processo decisório são relevantes, pois, apesar deste constituir-se em um fenômeno individual, possui certa complexidade, uma vez que seus efeitos se estendem por todos os sistemas sociais. No entanto, como afirma Germain (2011), existe ainda uma falta de clareza na definição do conceito especialização e, consequentemente, na diferenciação entre especialistas e novatos.

Considerando a relevância e complexidade do tema, há uma preocupação na literatura sobre a mensuração do nível de conhecimento especializado, sendo apontadas certas limitações no uso dos métodos tradicionais para mensurar tais níveis (WEISS et al., 2006; WITTEMANN et al., 2012). Diante deste contexto, Shanteau et al. (2003) propõe o método CWS (Cochran, Weiss e Shanteau), incluindo as variáveis discriminação e inconsistência, como alternativa complementar para mensuração do nível de conhecimento especializado. O índice CWS tem sido utilizado, principalmente em pesquisas internacionais sobre o tema, sendo comprovada sua eficiência (WEISS et al., 2006; PAULEY et al., 2009; WITTEMANN et al., 2012).

No entanto, mesmo havendo consenso acerca da relevância da temática, verifica-se, principalmente no contexto nacional, uma carência de investigações que abordem diferentes níveis de conhecimento no processo decisório, considerando a discriminação e a consistência no julgamento dos decisores (LÖBLER e HOPPEN, 2008). Dessa forma, realizar investigações neste campo de análise é de suma importância pela contribuição que podem ocasionar. Neste sentido, diferenciar indivíduos através do seu nível de experiência acerca do assunto objeto de decisão para verificar diferentes atitudes diante de determinado fato, e, por meio destas, explicar variáveis comportamentais é importante fonte de pesquisas em estudos organizacionais (LÖBLER e HOPPEN, 2008).

Diante da relevância do tema, visando contribuir para ampliar os estudos no contexto nacional, o objetivo geral deste trabalho consiste em avaliar a consistência e a discriminação de indivíduos com diferentes níveis de conhecimento no processo decisório, considerando a complexidade da tarefa. Frente a esta colocação, os objetivos específicos consistem em: (i) mensurar, por meio do índice CWS, o nível de conhecimento de indivíduos especialistas e não especialistas e; (ii) identificar se indivíduos com diferentes níveis de conhecimento apresentam maior discriminação e consistência no processo decisório.

Na próxima seção, são abordados os pressupostos teóricos relacionados aos níveis de conhecimento do decisor e mensuração dos níveis de conhecimento. Na seção seguinte, são descritos os aspectos metodológicos, relacionados à realização da pesquisa, para, posteriormente, (seção quatro) 
apresentar os resultados encontrados. Por fim, na seção cinco, são apresentadas considerações finais acerca do estudo, limitações e sugestões para pesquisas futuras.

\section{Referencial teórico}

Nesta seção, apresenta-se uma das formas de diferenciar os indivíduos diante de contextos de tomada de decisão, neste caso, o seu conhecimento acerca do assunto em questão. Para tanto, são abordados primeiramente os fundamentos teóricos sobre níveis de conhecimento do decisor e posteriormente sobre a mensuração dos níveis de conhecimento.

\subsection{Níveis de conhecimento do decisor}

O nível de conhecimento dos decisores possui grande relevância na compreensão de comportamentos decisórios (LÖBLER e HOPPEN, 2008). Conforme Malhotra et al. (2007), a qualidade da decisão está diretamente relacionada ao nível de conhecimento dos decisores sobre o assunto. No entanto, apesar de sua significativa importância no processo decisório, a diferenciação entre indivíduos, considerando seu conhecimento específico, consiste em uma tarefa complexa, sendo abordada a partir de uma amplitude de aspectos por diferentes autores.

Para Patel e Groen (1991), a classificação de indivíduos de acordo com o nível de conhecimento pode ser feita através de um continuum, dividido em categorias, variando de baixo nível de conhecimento (indivíduos que possuem apenas o senso comum ou um conhecimento do dia a dia sobre determinado assunto) a alto nível de conhecimento (indivíduos que possuem amplo domínio de determinado assunto). Já Bedard et al. (1993) advogam que o alto nível de conhecimento pode ser expresso como uma habilidade adquirida pela prática, que proporciona um bom desempenho qualitativo em uma tarefa de domínio específico. Dessa forma, um indivíduo com alto nível de conhecimento possui um amplo conhecimento e habilidades de procedimento (BEDARD et al. 1993).

Nesse sentido, Löbler e Hoppen (2008) destacam que, em diferentes tarefas decisórias, encontram-se indivíduos que detêm um nível diferenciado de conhecimento relativo ao objeto de decisão. Quando há um conhecimento relativamente alto de um em relação ao outro, o primeiro é reconhecido como especialista (expert), enquanto o outro é considerado como novato (novice).

Sob este aspecto, Ericsson (2006) afirma que a especialização refere-se a características, habilidades e conhecimentos que distinguem especialistas de novatos e pessoas menos experientes. Chen et al. (2006) ao analisar a perspectiva de diferentes autores sobre a diferenciação entre especialistas e novatos constataram que as distinções são abordadas a partir de alguns enfoques. $\mathrm{O}$ primeiro deles está associado a diferenças entre especialistas e novatos, considerando a formação e a experiência na área de investigação (SIMMONS e LUNETTA, 1993; CHEN et al., 2006).

Em relação à experiência, Markman e Medin (2001) destacam que esta pode interferir no processo de decisão, influenciando o tomador de decisão através da maneira como este percebe determinado assunto, permitindo que o decisor vá além da informação disponível e até mesmo, impedindo o processo de decisão. Ainda, para Markman e Medin (2001), especialistas podem utilizar o conhecimento prévio para influenciar uma escolha, podendo repetir esta escolha ao invés de comparar uma série de opções.

O segundo enfoque destacado por Chen et al. (2006) consiste na organização de estruturas conceituais, sendo que os especialistas possuem uma melhor representação mental (estrutura hierárquica organizada) dos conceitos de domínio (SPIRES e DONLEY, 1998), quando comparados aos novatos. Já o terceiro, refere-se à diferenciação entre ambos, associada às estratégias de busca por informações adotadas, sendo que os especialistas priorizam estratégias de localização de informações em profundidade, enquanto os novatos demonstram preferência por estratégias de amplitude de informações (JENKINS et al., 2003).

Tais perspectivas indicam diferenças no processamento das informações e na decisão de especialistas e novatos, sendo foco de diversos estudos (CHASE e SIMON, 1973; LÖBLER e HOPPEN, 2008; GERMAIN, 2011). A Tabela 1 ilustra a comparação de características de indivíduos especialistas e novatos feita por Sternberg (2000) e Chen et al. (2006) para resolução de problemas.

Percebe-se que o interesse dos autores em compreender e distinguir as características de especialistas e novatos perpassa a classificação e descrição de tais diferenças, congregando aspectos 
mais amplos, associados a estratégias de aprendizagem, à cognição e ao comportamento humano. Ao analisar a diversidade de abordagens que buscam compreender aspectos relacionados à especialização, Germain (2011) constatou sua amplitude e evolução. Essas inicialmente focavam o processamento da informação e a inteligência artificial (1940-1970), avançando, ao longo dos anos, para aspectos relacionados à memória e à resolução de problemas (1980-1990), à inteligência emocional e a formas de especialização (a partir de 1990).

Tabela 1 - Diferenças de características de especialistas e novatos na resolução de problemas e características de aprendizagem

\begin{tabular}{|c|c|c|}
\hline Foco de comparação & Especialistas & Novatos \\
\hline Esquemas & $\begin{array}{l}\text { Possuem grandes e ricos esquemas } \\
\text { que contêm muito conhecimento } \\
\text { declarativo sobre o domínio. }\end{array}$ & $\begin{array}{l}\text { Possuem esquemas relativamente } \\
\text { empobrecidos que contêm relativamente } \\
\text { menos conhecimento sobre o assunto. }\end{array}$ \\
\hline Unidades de conhecimento & $\begin{array}{l}\text { Possuem unidades de conhecimento } \\
\text { bem organizadas e altamente } \\
\text { interconectadas nos esquemas. }\end{array}$ & $\begin{array}{l}\text { Possuem unidades de conhecimento } \\
\text { dispersas, pobremente organizadas e } \\
\text { interconectadas. }\end{array}$ \\
\hline Dedicação de tempo & $\begin{array}{l}\text { Despendem proporcionalmente mais } \\
\text { tempo determinando como } \\
\text { representar um problema do que na } \\
\text { procura e na execução da estratégia } \\
\text { do mesmo. }\end{array}$ & $\begin{array}{l}\text { Despendem proporcionalmente mais } \\
\text { tempo, procurando a estratégia de um } \\
\text { problema, executando-a em vez de } \\
\text { determinar como representá- lo. }\end{array}$ \\
\hline Modelos mentais & Possuem modelos mentais globais. & Possuem modelos mentais locais. \\
\hline Simulação mental & $\begin{array}{l}\text { Simulação mental de funções } \\
\text { integradas. }\end{array}$ & Simulação mental de funções isoladas. \\
\hline Primeiras estratégias & $\begin{array}{l}\text { Profundidade nas } \\
\text { estratégias utilizadas. }\end{array}$ & $\begin{array}{l}\text { Amplitude nas primeiras estratégias } \\
\text { utilizadas. }\end{array}$ \\
\hline
\end{tabular}

Fonte: Adapatdo de Sternberg (2000) e Chen et al. (2006).

Tal evolução contribuiu significativamente para ampliar as discussões acerca da complexidade e relevância do tema. Nesse sentido, segundo Germain (2011), para melhor compreender a especialização e utilizá-la para mensurar o desempenho humano, torna-se necessária uma ampliação dos estudos sobre o tema.

\subsection{Mensuração dos níveis de conhecimento}

A avaliação de níveis de conhecimento e a mensuração dos níveis de especialização dos indivíduos têm despertado algumas discussões nos estudos sobre processo decisório. As formas tradicionais de identificação de especialização, como por exemplo, o grau de experiência do decisor e sua certificação, a aclamação social e os testes de conhecimento têm sido questionados em função de suas limitações.

Sob este aspecto, Shanteau et al. (2003) destacam que os modos tradicionais de identificação de especialização possuem algumas fragilidades, relacionadas, principalmente, à subjetividade e à ausência de parâmetros concretos. Exemplificando tal constatação, Wittemann et al. (2012) afirmam que alguns estudos têm demonstrado que o aumento da experiência nem sempre está associado a melhores julgamentos, sugerindo que este critério tradicional, isoladamente, pode não ser suficiente para mensurar o nível de especialização.

Shanteau et al. (2003) ampliaram as perspectivas acerca da mensuração de experiência, ponderando a discriminação e a consistência, ao invés de considerar somente a capacidade e os critérios externos (WEISS et al., 2006). Segundo Löbler e Hoppen (2008), os autores Shanteau et al. (2003) exploraram uma forma de mensuração que combina modelos tradicionais, qualitativos, com um novo modelo, mais quantitativo, denominado de índice CWS (Cochran-Weiss-Shanteau índex). Esse índice combina duas medidas, discriminação e consistência, cada uma das quais é uma condição necessária, mas não suficiente, isoladamente, para avaliar um especialista (PAULEY et al., 2009). Como afirmam Wittemann et al. (2012), o índice CWS avalia a capacidade de o indivíduo discriminar de forma consistente. 
Assim, Shanteau et al. (2003) argumentaram que a discriminação, associada à capacidade de diferenciar entre casos semelhantes, mas não idênticos, é uma forte característica dos especialistas. Da mesma forma, baseando-se nos estudos de Einhorn (1974), os referidos autores advogam que a consistência, relacionada à capacidade de repetir um julgamento em uma situação similar, também é essencial para avaliar um especialista. Para Shanteau et al. (2003), se um indivíduo não consegue repetir seu julgamento em uma situação similar, pode-se afirmar que este possui inconsistência.

Valendo-se de uma formulação utilizada por Cochran (1943), os conceitos de discriminação e inconsistência foram combinados usando uma proporção. Cabe acrescentar que, a mensuração da discriminação se dá a partir do cálculo da variância das médias em cada estímulo para cada indivíduo e, a mensuração da inconsistência se dá a partir da média das variâncias para o mesmo estímulo considerando a média entre estímulos (WEISS e SHANTEAU, 2013). A medida de discriminação (quanto mais, melhor) é dividida pela medida da inconsistência (quanto menor, melhor), assim:

$$
\text { CWS }=\frac{\text { Discriminação }}{\text { Inconsistência }}
$$

O índice CWS, proposto por Shanteau et al. (2003), supõe que, quanto maior a discriminação e maior consistência das respostas, maior será o índice, portanto, o respondente será considerado com um maior nível de conhecimento. Como afirma Löbler (2005), um aspecto lembrado pelos autores é que o uso da proporção é descritiva, não inferencial, isto é, o CWS é mais qualitativo do que quantitativo. Como não existem comparações estatísticas entre dois índices, não pode ser determinado o nível de significância. O índice CWS é utilizado para determinar se um indivíduo possui maior (alto valor) ou menor (baixo valor) especialização. Ainda, Shanteau et al. (2003) sugerem que o índice, não deve ser utilizado de maneira aleatória, e sim como suplementação de outras abordagens, uma vez que deve haver uma separação prévia do conjunto de pessoas que irá participar da aplicação do método.

Diversos autores têm utilizado o índice CWS em suas pesquisas, principalmente no contexto internacional (WEISS et al., 2006; PAULEY et al., 2009; WITTEMANN et al., 2012), sendo este considerado uma valiosa ferramenta nos estudos que tangenciem aspectos relacionados à especialização, corroborando sua aplicação na mensuração de níveis de conhecimento. Weiss et al. (2006) demonstraram o valor do índice em uma análise de decisões de priorização feitas por estudantes de terapia ocupacional, antes e depois, receberam um treinamento específico. As pontuações do CWS dos alunos melhoraram consideravelmente após o treinamento. Pauley et al. (2009), a fim de medir o conhecimento em aeronáutica sobre a percepção de risco relacionado ao clima, realizaram dois estudos utilizando o índice. No primeiro estudo não foi encontrada relação entre a experiência de voo e as pontuações CWS. No segundo, onde a tarefa para condução da pesquisa fora modificada, a fim de envolver uma tarefa de bloqueio, houve uma relação entre a experiência de voo e as pontuações CWS. Wittemann et al (2012) aplicaram o índice no campo de avaliação clínica, encontrando nítida diferença para o CWS com relação ao nível de conhecimento para os grupos pesquisados.

\section{Procedimentos Metodológicos}

Esta seção descreve os procedimentos metodológicos que permitiram a realização deste estudo. Dessa forma, essa seção está divida em cinco partes, as quais envolvem o modelo de pesquisa, a tarefa decisória, a validação da tarefa, os sujeitos experimentais e a condução do experimento.

\subsection{Modelo de pesquisa}

O presente estudo fundamentou-se por meio da pesquisa experimental, que de acordo Aaker, Kumar e Day (2004), que consiste numa situação em que se manipulam uma ou mais variáveis independentes e mede seu efeito sobre uma ou mais variáveis dependentes. Ainda, no entender de Cozby (2003), a pesquisa experimental busca eliminar a influência de variáveis estranhas, mantendose todas as características do ambiente constantes, exceto as variáveis manipuladas. Como variáveis independentes, têm-se o nível de conhecimento do decisor e a complexidade da tarefa e, como variável dependente, o resultado da decisão. A Figura 1 apresenta o modelo da pesquisa. 


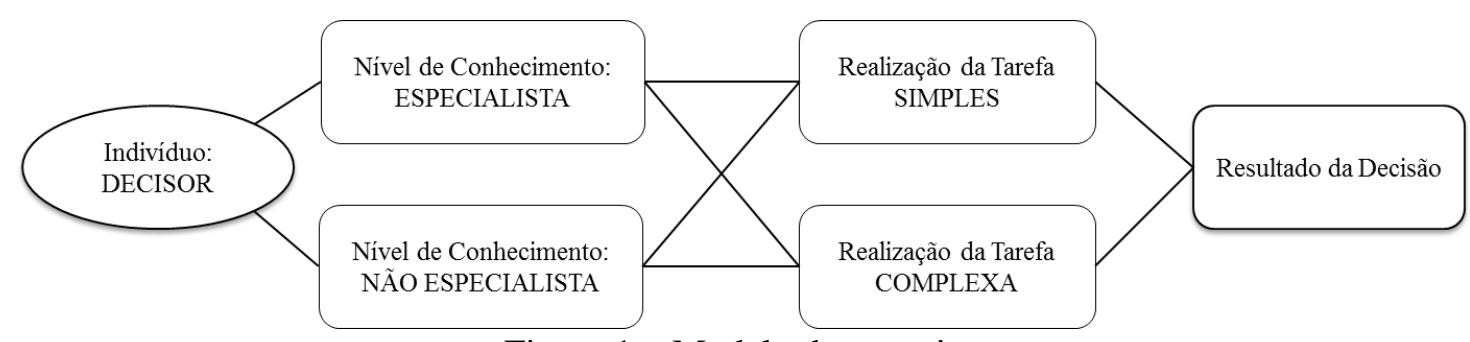

Figura 1 - Modelo de pesquisa

Com relação à classificação do nível de conhecimento do decisor, foram consideradas as distinções propostas por Stenberg (2000) e Chen et al. (2006) para definir especialistas e não especialistas, bem como os critérios experiência e formação profissional na área. No que se refere à complexidade da tarefa, de acordo com Fisher et al. (2003), a classificação de tarefa simples e de complexa foi definida como uma relação do número de células num espaço decisório, contadas a partir de uma matriz, relacionando escolhas (número de alternativas) e critérios de decisão (número de atributos). Nesse contexto, para esses autores, uma tarefa com vinte células pode ser considerada simples, enquanto que tarefas complexas correspondem àquelas que possuem quarenta ou mais células, relacionando atributos e alternativas.

\subsection{Tarefa decisória}

A tarefa decisória está relacionada ao segmento imobiliário e consiste na simulação de compra de um imóvel residencial (casa própria/apartamento). A tarefa simples foi definida por três alternativas de apartamentos disponíveis para a compra (Marselha, Piazza Navona e Hamburgo) e a tarefa complexa por seis opções (Veneza, Lisboa, Dover, Marselha, Piazza Navona e Hamburgo), sendo que três eram repetidas. A fim de evitar que o decisor repetisse a tarefa simples, foi realizada uma modificação na tarefa decisória. Inicialmente o indivíduo realizava a tarefa simples e, na sequência, a complexa. Todos os apartamentos foram caracterizados como: na planta, com prazo de dois anos para entrega, localizados na mesma cidade, com três dormitórios, uma garagem, elevador, salão de festas e churrasqueira.

Além desses critérios, presentes em todas as alternativas, foram utilizados: preço, localização, área privativa, padrão de acabamento, posição solar, posição em relação ao andar, posição do apartamento no prédio e número de banheiros, como importantes no processo de escolha do imóvel. Esses critérios foram definidos com base em Oliveira et al. (2008); manuais de sindicatos do ramo imobiliário - Sindicato das Empresas de Compra, Venda, Locação e Administração de Imóveis Comerciais e Residenciais de São Paulo (SICOVI-SP) e por especialistas na área, corretores de imóveis com mais de dez anos de experiência no segmento.

Para a execução da tarefa, foi disponibilizado ao indivíduo um Sistema de Apoio à Decisão (SAD), denominado DECISOR (Figura 2), baseado no método de Pontuação Direta, no qual se solicita ao usuário atribuir pesos a cada um dos critérios que representarão a importância relativa dos mesmos. O método de pontuação direta prevê que seja construído um conjunto de níveis de impacto, ordenados preferencialmente, definindo o pior e o melhor nível. A esses dois níveis são associados dois valores que servirão de âncora para a escala, normalmente 0 e 100 , respectivamente o pior e o melhor (LÖBLER, 2005). 


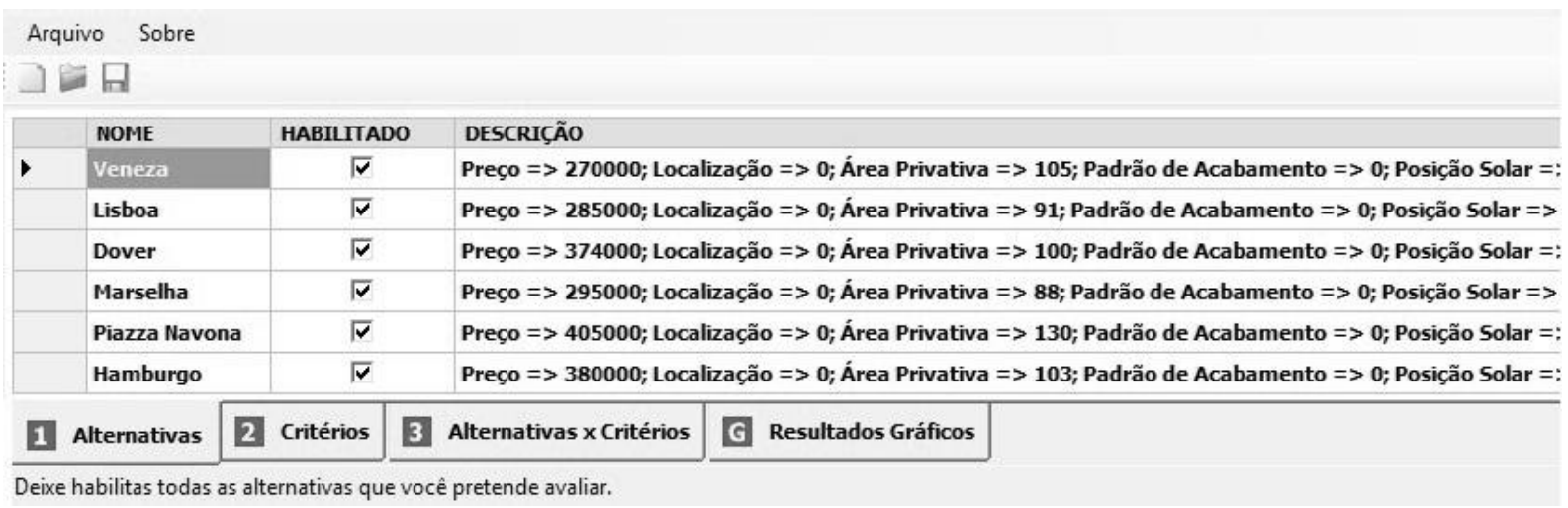

Figura 2 - Tela da tarefa do DECISOR - alternativas

A tela inicial do DECISOR apresenta todas as alternativas habilitadas disponíveis para escolha. A partir da sua visualização, o indivíduo tem a opção de desabilitar algumas das alternativas, caso opte por não trabalhar com todas elas.

O sistema funciona com atribuição de pesos, onde deverá ser atribuído inicialmente o peso aos critérios (preço, localização, área privativa, padrão de acabamento, posição solar, posição em relação ao andar, posição do apartamento no prédio e número de banheiros) e posteriormente dentro do próprio critério, considerando cada uma das alternativas. A Figura 3 apresenta a terceira tela do DECISOR, exemplo de atribuição de pesos do critério preço para cada alternativa disponível no sistema.

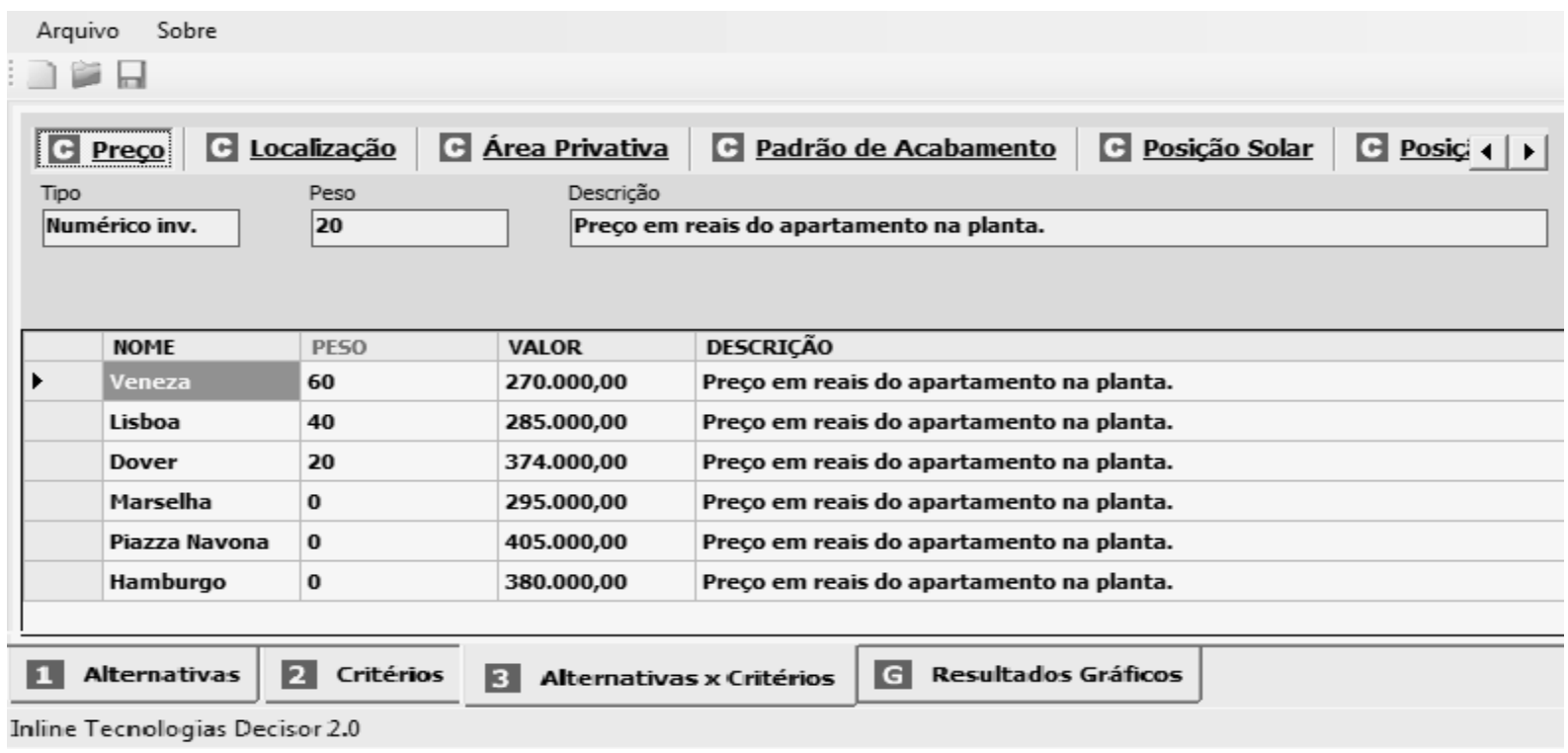

Figura 3 - Tela da tarefa do DECISOR - alternativas x critérios

Além do sistema DECISOR, os indivíduos também recebiam para a realização da tarefa um material ilustrativo (folders) com as imagens de imóveis hipotéticos e a descrição dos critérios. A Figura 4 apresenta o layout de um dos folders utilizados nesse estudo. 


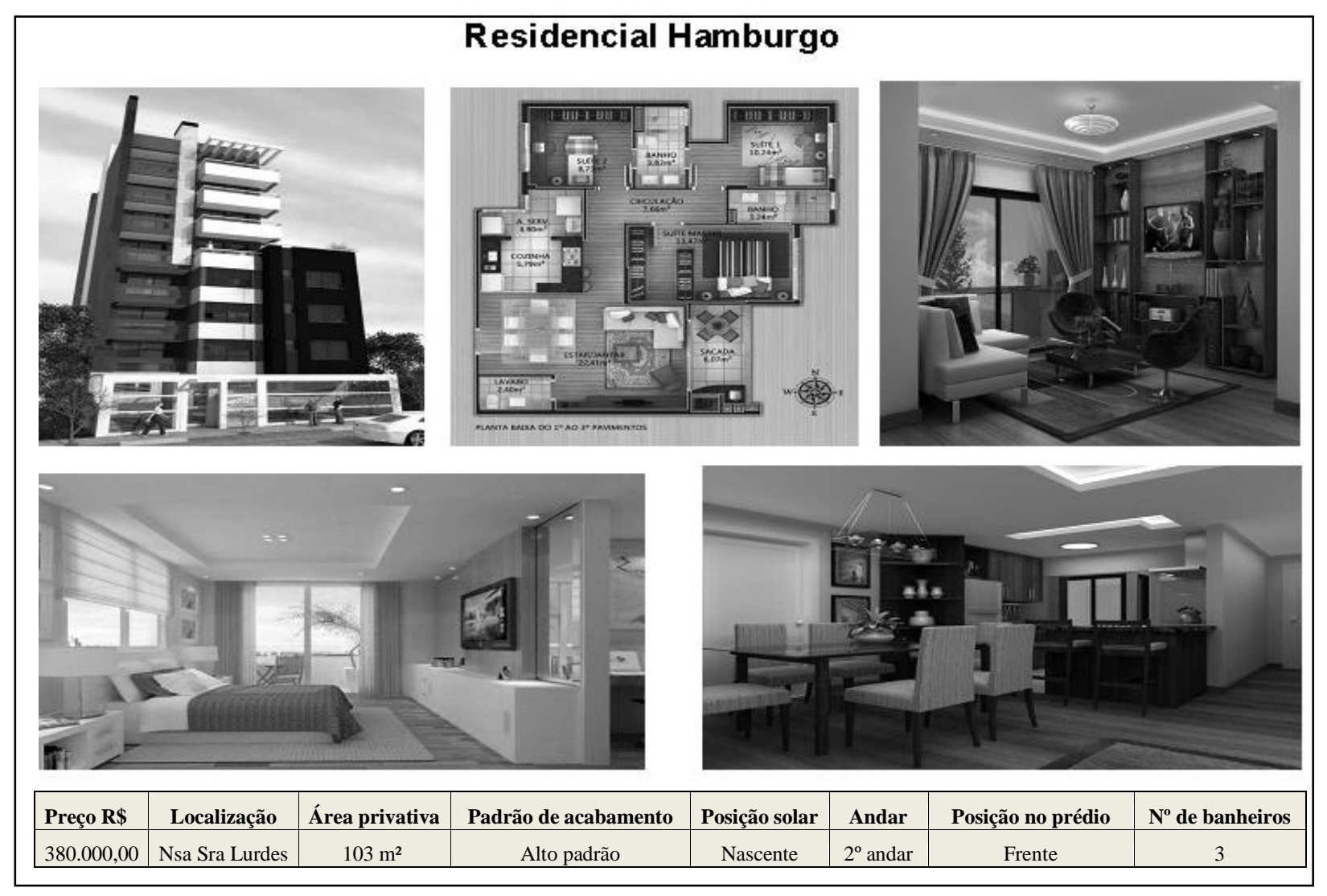

Figura 4 - Folder ilustrativo da alternativa de apartamento Hamburgo Fonte: Adaptado de Imofar imobiliária

\subsection{Validação da tarefa}

A tarefa experimental passou por um processo de validação, no qual três sujeitos experimentais participaram de um pré-teste. Cabe acrescentar que o software DECISOR, utilizado neste experimento, já havia sido validado em estudo anterior por Löbler (2005).

Durante a aplicação do pré-teste, buscando levantar informações pertinentes quanto aos ajustes a serem realizados na aplicação da tarefa, foram constatadas questões que deveriam ser alteradas para facilitar o processo de aplicação. Primeiramente procedeu-se a alteração no layout dos folders, a fim de facilitar a leitura dos critérios e observação das imagens de cada alternativa de apartamento. Observou-se também que a entrega dos folders aos decisores deveria ser posterior à atribuição de pesos aos critérios no sistema. Esse procedimento evitaria a escolha prévia do apartamento somente pela observação das imagens constante nos folders.

\subsection{Sujeitos experimentais}

Participaram da pesquisa, como sujeitos experimentais, indivíduos que possuem conhecimento sobre o objeto de decisão (imóvel residencial). Assim, foram selecionados doze sujeitos, denominados especialistas, ou seja, corretores de imóveis registrados há mais de cinco anos no Conselho Regional de Corretores de Imóveis (CRECI) e doze, denominados não especialistas, os quais possuíam pósgraduação e haviam adquirido algum imóvel (casa própria/apartamento) recentemente ou que estavam pesquisando algum imóvel para compra. Dessa forma, utilizou-se o critério de conhecimento do objeto para a seleção e classificação dos sujeitos que, conforme Löbler (2005), é uma variável influenciadora. Ainda, em relação à classificação das pessoas não especialistas, adotou-se o critério proposto por Shanteau et al. (2003) de que indivíduos que possuem pós-graduação (em áreas afins ao objeto de estudo) podem ser considerados novatos (neste estudo denominados de não especialistas) em tomada de decisão. 
O experimento foi administrado individualmente com cada sujeito, em um laboratório, com auxílio de um computador. Cabe acrescentar que as tarefas foram aplicadas ao mesmo grupo experimental, ou seja, as mesmas pessoas participaram das duas condições, executando, primeiramente, a tarefa simples e, posteriormente, a tarefa complexa. Neste caso, conforme Cozby (2003) tem-se um delineamento com medidas repetidas, no qual os mesmos indivíduos participam das duas condições, passando por repetidas mensurações da variável dependente, após cada condição experimental.

\subsection{Condução do experimento}

Para a realização do experimento, primeiramente foi realizada uma breve apresentação, por meio de slides, sobre o objetivo do estudo, aplicação da tarefa decisória e uso do DECISOR. Em seguida, era entregue ao indivíduo uma cópia das instruções para realização da tarefa, sendo que o pesquisador procedia a explicação de cada etapa da mesma e como deveria ser realizada com o apoio do sistema. Após estes procedimentos, o indivíduo era informado de que a realização do experimento iniciaria com a resolução da tarefa simples e, após, da complexa e que ambas não tinham limite de tempo, ou seja, poderiam ser executadas no tempo que o sujeito experimental julgasse necessário.

Tendo o indivíduo iniciado a tarefa, após a atribuição de pesos aos critérios, o pesquisador lhe entregava os folders com as imagens das três alternativas de apartamentos constantes na tarefa simples. Esse material servia como apoio ao processo de escolha na tarefa decisória. Ao final da tarefa simples, o sujeito experimental avisava o pesquisador da sua conclusão, o qual recolhia o material de apoio (folders) e, na sequência, preparava o sistema para aplicação da tarefa complexa.

Para a realização da tarefa complexa, após a atribuição de pesos aos critérios, o sujeito experimental recebia novamente os folders utilizados na tarefa simples e mais três alternativas para fins de realização da tarefa complexa. Após a conclusão da tarefa complexa, o sujeito experimental respondia um questionário sobre questões relativas à concordância ou não com a escolha, possíveis dificuldades e contribuições do sistema no processo de escolha. A Figura 5 ilustra as etapas da realização do experimento. Cabe salientar que os procedimentos adotados na aplicação da tarefa ocorreram de forma idêntica, tanto para os decisores considerados especialistas, quanto para os decisores considerados não especialistas.

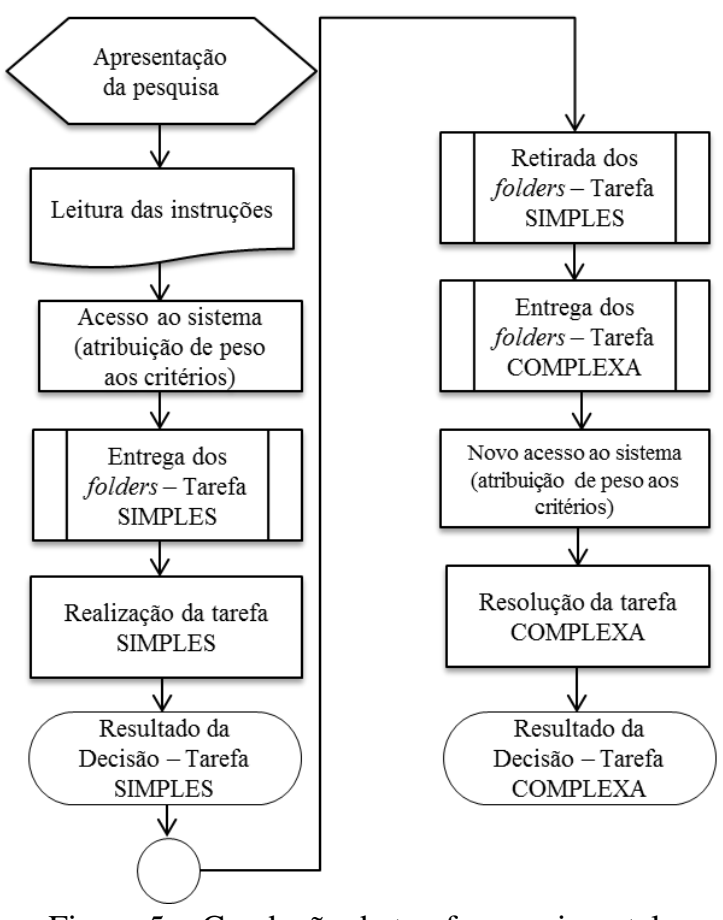

Figura 5 - Condução da tarefa experimental 
Os dados das duas tarefas decisórias foram quantitativamente analisados, com o auxílio do software "Microsoft Office Excel ${ }^{\circledR}$ ". Dessa forma, procedeu-se aos cálculos para mensuração do índice CWS, a fim de avaliar a consistência e a discriminação de indivíduos especialistas e não especialistas no processo decisório, considerando a complexidade da tarefa. Cabe mencionar que, nesse estudo, as variáveis utilizadas para o cálculo do CWS foram a atribuição de pesos aos critérios (preço, localização, área privativa, padrão de acabamento, posição solar, posição em relação ao andar, posição do apartamento no prédio e número de banheiros) e o resultado da decisão (alternativas/apartamentos).

\section{Análise dos resultados}

Esta seção abrange os resultados encontrados no estudo e está organizada em dois tópicos. Primeiramente é exposta uma descrição dos indivíduos que participaram do experimento e, posteriormente, é apresentada a mensuração e a análise do índice CWS para indivíduos com diferentes níveis de conhecimento.

\subsection{Perfil dos sujeitos experimentais}

Participaram deste estudo 24 indivíduos, sendo 12 especialistas e 12 não especialistas. Constatou-se que, entre os indivíduos especialistas, a média de idade configurou-se em 42 anos, sendo $66,7 \%$ do sexo masculino e 33,3 \% do sexo feminino. Destes, 41,7\% são casados, 33,3\% são solteiros e $25 \%$ são separados. Quanto ao nível de escolaridade, $66 \%$ possuem ensino superior e $17 \%$ possuem pós-graduação e ensino médio. Em relação à leitura de revistas especializadas sobre imóveis, $75 \%$ afirmou ler regularmente e $16,7 \%$ somente esporadicamente. Considerando o tempo de registro no CRECI, a média configurou-se em 12,42 anos e o tempo de atuação como corretores de imóveis foi de 13,92 anos.

Quanto aos indivíduos não especialistas, a média de idade foi de 34,08 anos, sendo $83,3 \%$ do sexo feminino e $16,7 \%$ do sexo masculino, predominando os indivíduos solteiros e que possuem união estável (41,7\% para ambos). Quanto ao nível de escolaridade, todos os indivíduos possuem pósgraduação. Em relação à leitura de revistas especializadas sobre imóveis, 25\% dos participantes afirmaram ler regularmente e 50\% esporadicamente. Em relação às atividades profissionais, todos são servidores públicos, atuantes em cargos de técnico-administrativos em educação de nível superior. Cabe salientar que as informações acerca do perfil dos indivíduos que participaram do estudo foram identificadas mediante questionário aplicado após a realização do experimento.

\subsection{Mensuração e análise do índice CWS para indivíduos com diferentes níveis de conhecimento}

Inicialmente, buscou-se mensurar o nível de conhecimento de indivíduos especialistas e não especialistas. Considerando a atribuição de peso aos critérios (A: preço; B: localização; C: área privativa; D: padrão de acabamento; E: posição solar; F: posição em relação ao andar; G: posição do apartamento no prédio e; $\mathrm{H}$ : número de banheiros) no processo de decisão da tarefa simples, cujo objetivo era a escolha de uma alternativa entre três apartamentos, e da tarefa complexa, a qual possuía seis alternativas de apartamentos, calculou-se o índice CWS, apresentado na Tabela 2.

Ao analisar a Tabela 2, onde são apresentados os pesos atribuídos aos critérios pelos decisores, considerados especialistas (E1 a E12) e não especialistas (NE13 a NE 24), observou-se que, de forma geral, os não especialistas obtiveram os maiores índices CWS. Como o índice CWS mede discriminação e inconsistência, pode-se inferir que os nãos especialistas discriminaram mais e foram menos inconsistentes na atribuição de peso aos critérios, ao analisarem as alternativas da tarefa simples e posteriormente da tarefa complexa. 
Tabela 2 - Atribuição de pesos aos critérios e CWS dos critérios

\begin{tabular}{|c|c|c|c|c|c|c|c|c|c|c|c|c|c|c|c|c|c|c|c|}
\hline \multirow{2}{*}{$\begin{array}{l}\text { Nível } \\
\text { conh./ } \\
\text { Decisor }\end{array}$} & \multicolumn{8}{|c|}{ Tarefa simples } & \multicolumn{8}{|c|}{ Tarefa complexa } & \multirow{2}{*}{$\begin{array}{l}\text { Discri- } \\
\text { minação }\end{array}$} & \multirow{2}{*}{$\begin{array}{l}\text { Incon- } \\
\text { sistência }\end{array}$} & \multirow[t]{2}{*}{ CWS } \\
\hline & $\bar{A}$ & $\mathrm{~B}$ & $\mathrm{C}$ & $\mathrm{D}$ & $\mathrm{E}$ & $\mathrm{F}$ & $\mathrm{G}$ & $\mathrm{H}$ & $\mathrm{A}$ & $\mathrm{B}$ & $\mathrm{C}$ & $\mathrm{D}$ & $\mathrm{E}$ & $\mathrm{F}$ & $\bar{G}$ & $\mathrm{H}$ & & & \\
\hline E12 & 15 & 30 & 10 & 10 & 10 & 10 & 5 & 10 & 20 & 30 & 10 & 10 & 10 & 10 & 5 & 5 & 125,00 & 3,13 & 40,00 \\
\hline E8 & 10 & 5 & 10 & 5 & 30 & 15 & 15 & 10 & 10 & 10 & 10 & 5 & 30 & 15 & 10 & 10 & 117,85 & 3,13 & 37,71 \\
\hline E2 & 0 & 30 & 15 & 20 & 15 & 5 & 10 & 5 & 5 & 30 & 10 & 20 & 15 & 10 & 5 & 5 & 164,28 & 6,25 & 26,29 \\
\hline E3 & 22 & 24 & 15 & 10 & 20 & 10 & 1 & 10 & 20 & 20 & 8 & 10 & 20 & 15 & 2 & 5 & 105,00 & 7,5 & 14,00 \\
\hline E11 & 40 & 30 & 5 & 0 & 10 & 5 & 5 & 5 & 30 & 30 & 0 & 10 & 30 & 0 & 0 & 0 & 378,57 & 43,75 & 8,65 \\
\hline E1 & 30 & 20 & 0 & 10 & 20 & 10 & 0 & 10 & 40 & 40 & 0 & 0 & 20 & 0 & 0 & 0 & 385,71 & 50,00 & 7,71 \\
\hline E10 & 24 & 30 & 5 & 20 & 10 & 5 & 1 & 5 & 20 & 15 & 10 & 20 & 20 & 5 & 5 & 5 & 23,88 & 5,75 & 5,75 \\
\hline $\mathrm{E} 4$ & 10 & 30 & 10 & 20 & 10 & 5 & 5 & 10 & 5 & 30 & 10 & 10 & 10 & 20 & 5 & 10 & 117,85 & 21,88 & 5,39 \\
\hline E7 & 20 & 15 & 15 & 10 & 15 & 10 & 5 & 10 & 20 & 10 & 15 & 15 & 10 & 10 & 10 & 10 & 28,57 & 6,25 & 4,57 \\
\hline E5 & 5 & 20 & 10 & 10 & 20 & 10 & 15 & 10 & 5 & 20 & 10 & 10 & 20 & 10 & 15 & 10 & 57,14 & 0 & 0 \\
\hline E6 & 60 & 10 & 10 & 5 & 0 & 5 & 0 & 0 & 60 & 10 & 10 & 5 & 0 & 5 & 0 & 0 & 810,71 & 0 & 0 \\
\hline E9 & 13 & 12 & 10 & 12 & 20 & 16 & 7 & 10 & 13 & 12 & 10 & 12 & 20 & 16 & 7 & 10 & 32,00 & 0 & 0 \\
\hline NE16 & 30 & 20 & 20 & 15 & 5 & 0 & 0 & 10 & 30 & 25 & 20 & 10 & 5 & 0 & 0 & 10 & 239,29 & 3,13 & 76,57 \\
\hline NE21 & 40 & 15 & 5 & 10 & 10 & 10 & 5 & 5 & 40 & 10 & 10 & 10 & 10 & 5 & 5 & 10 & 257,14 & 6,25 & 41,14 \\
\hline NE14 & 20 & 20 & 10 & 10 & 15 & 15 & 5 & 5 & 20 & 20 & 10 & 10 & 20 & 10 & 5 & 5 & 75,00 & 3,13 & 24,00 \\
\hline NE22 & 20 & 20 & 15 & 15 & 5 & 15 & 5 & 5 & 20 & 20 & 15 & 15 & 5 & 10 & 5 & 10 & 75,00 & 3,13 & 24,00 \\
\hline NE24 & 30 & 40 & 5 & 5 & 10 & 5 & 3 & 2 & 20 & 50 & 10 & 10 & 3 & 2 & 4 & 1 & 451,86 & 19,38 & 23,32 \\
\hline NE20 & 20 & 15 & 20 & 5 & 15 & 10 & 5 & 10 & 15 & 20 & 20 & 5 & 15 & 10 & 5 & 10 & 67,86 & 3,13 & 21,71 \\
\hline NE15 & 25 & 30 & 20 & 5 & 5 & 5 & 5 & 5 & 20 & 30 & 15 & 15 & 5 & 5 & 5 & 5 & 189,29 & 9,38 & 20,19 \\
\hline NE17 & 15 & 30 & 0 & 20 & 20 & 5 & 5 & 5 & 30 & 15 & 0 & 20 & 20 & 5 & 5 & 5 & 182,14 & 28,13 & 6,48 \\
\hline NE13 & 15 & 10 & 10 & 10 & 15 & 10 & 15 & 15 & 20 & 15 & 15 & 10 & 10 & 10 & 10 & 10 & 10,71 & 9,38 & 1,14 \\
\hline NE18 & 20 & 20 & 15 & 20 & 15 & 0 & 5 & 5 & 20 & 20 & 15 & 20 & 15 & 0 & 5 & 5 & 128,57 & 0 & 0 \\
\hline NE19 & 30 & 50 & 10 & 10 & 0 & 0 & 0 & 0 & 30 & 50 & 10 & 10 & 0 & 0 & 0 & 0 & 671,43 & 0 & 0 \\
\hline NE23 & 20 & 20 & 15 & 15 & 5 & 0 & 5 & 20 & 20 & 20 & 15 & 15 & 5 & 0 & 5 & 20 & 128,57 & 0 & 0 \\
\hline
\end{tabular}

Visando ilustrar graficamente a ponderação de pesos atribuída aos critérios, apresenta-se, na Figura 6, a decisão de um especialista (E12) e de um não especialista (NE16). Cabe acrescentar que a discussão dos dados, oriundos desses sujeitos experimentais, se justifica pelo fato dos mesmos apresentarem o maior índice CWS entre os participantes do estudo no que se refere a atribuição de pesos aos critérios.

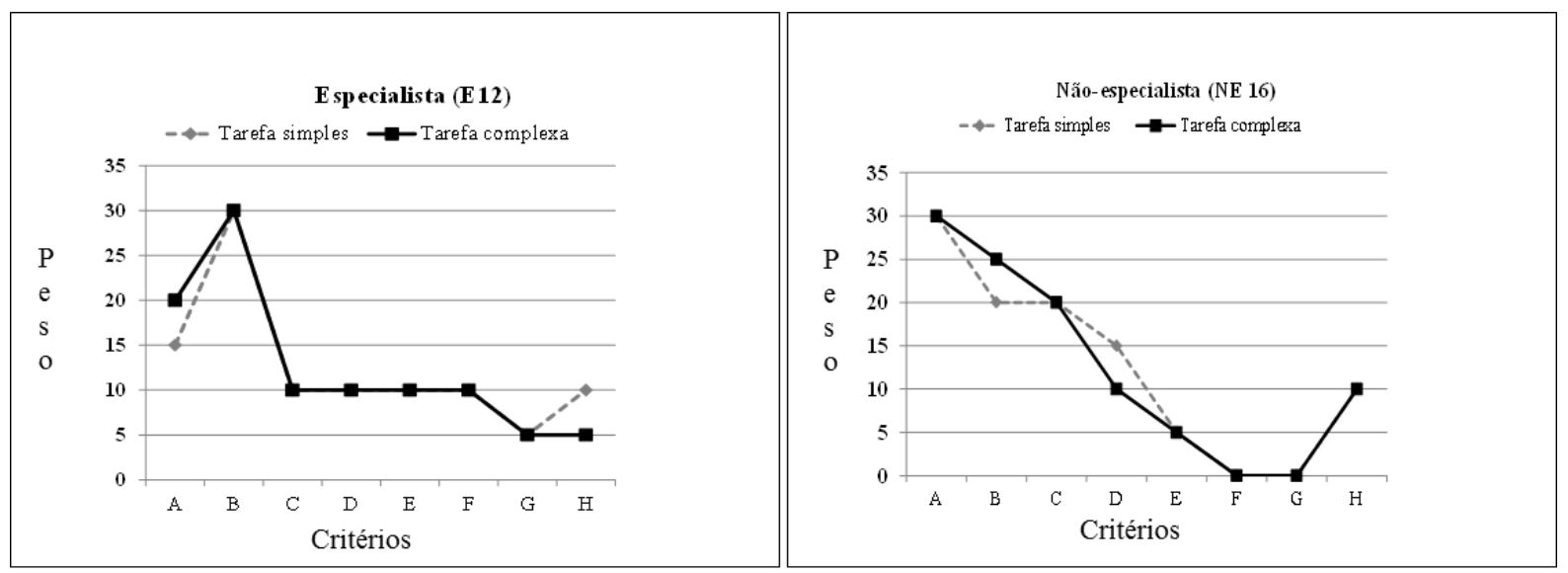

Figura 6 - Atribuição de pesos aos critérios do especialista (E12) e não especialista (NE16)

Fonte: dados da pesquisa

Na Figura 6, embora seja apenas ilustração de um decisor de cada grupo, o não especialista, além de discriminar mais, também apresenta maior consistência na decisão. A discriminação do não 
especialista foi de 239,29 , enquanto que do especialista foi de 125,00 . No cálculo da inconsistência, ambos foram mais consistentes, quando comparados aos demais decisores, apresentando um índice de inconsistência de 3,13. O índice CWS dos dois indivíduos representados no gráfico (E12 e NE16) foi de 40,00 e 76,57, respectivamente.

Nesse contexto, chama-se a atenção para o fato do índice CWS ser limitado, uma vez que os próprios autores do método, Shanteau et al. (2003) e também Malhotra et al. (2007) lembram que o índice é uma forma de mensuração que, se não for acompanhada de um método qualitativo externo, corre o risco de classificar como especialista um indivíduo que possui características pessoais de diferenciar alternativas. Assim, a consistência pode ser inerente à capacidade de o indivíduo lembrar, em tarefas sequenciais, os pesos atribuídos e refazer o caminho similar.

$\mathrm{Na}$ atribuição de pesos aos critérios, para o cálculo da média dos índices CWS dos diferentes decisores, considerou-se o somatório da raiz quadrada de cada um dos índices CWS divido pelo número de decisores, sendo este resultado elevado ao quadrado, conforme determina Shanteau et al. (2003). Assim, o valor médio para discriminação dos não especialistas foi de 206,40 e inconsistência de 7,08, enquanto a média do CWS para o grupo foi de 12,57. Por outro lado, para os especialistas, o valor médio para discriminação foi de 195,54 e inconsistência 12,20, sendo a média do CWS 7,99.

Quanto à decisão dos indivíduos em relação à escolha dos apartamentos, tanto na tarefa simples quanto na tarefa complexa, buscou-se mensurar o nível de conhecimento dos indivíduos especialistas e não especialistas, considerando três e seis alternativas, respectivamente. Os indivíduos inicialmente atribuíram pesos a cada um dos critérios (preço, localização, área privativa, padrão de acabamento, posição solar, posição solar, posição em relação ao andar, posição do apartamento no prédio e número de banheiros), para, posteriormente, avaliar cada uma das alternativas (apartamentos), considerando cada critério individualmente.

Ressalta-se que, em relação à tarefa complexa, os resultados apresentados na Tabela 3 foram normalizados, considerando os pesos atribuídos às três alternativas comuns às duas tarefas (Marselha, Piazza Navona e Hamburgo), excluindo-se da análise as demais alternativas (Veneza, Lisboa, Dover), que foram utilizadas apenas para diferenciar as duas tarefas. Na Tabela 3, são apresentadas as ponderações finais atribuídas a cada uma das alternativas, nas duas tarefas, bem como a discriminação, inconsistência e índice CWS de cada indivíduo.

Pode-se observar, através da Tabela 3 , que as ponderações atribuídas às alternativas (A: Marselha; B: Piazza Navona e; C: Hamburgo) pelos decisores considerados especialistas (E1 a E12) e não especialistas (NE13 a NE 24), também resultaram em um índice CWS superior à maioria dos não especialistas, evidenciando que estes discriminaram mais e foram mais consistentes ao ponderar as alternativas nas duas tarefas realizadas.

Tabela 3 - Decisão de especialistas e não especialistas e CWS da decisão

\begin{tabular}{|c|c|c|c|c|c|c|c|c|c|}
\hline \multirow{2}{*}{$\begin{array}{c}\text { Nível } \\
\text { conh./Decisor }\end{array}$} & \multicolumn{3}{|c|}{ Tarefa simples } & \multicolumn{3}{|c|}{ Tarefa complexa } & \multirow{2}{*}{$\begin{array}{l}\text { Discrimi- } \\
\text { nação }\end{array}$} & \multirow{2}{*}{$\begin{array}{c}\text { Incosis- } \\
\text { tência }\end{array}$} & \multirow{2}{*}{ CWS } \\
\hline & A & $\mathrm{B}$ & $\mathrm{C}$ & $\bar{A}$ & $\mathrm{C}$ & $\mathrm{C}$ & & & \\
\hline E6 & 37 & 100 & 51 & 34 & 100 & 54 & 2217,91 & 3,57 & 621,55 \\
\hline E2 & 35 & 45 & 100 & 25 & 36 & 100 & 2855,49 & 31,62 & 90,32 \\
\hline E4 & 50 & 56 & 100 & 55 & 66 & 100 & 1269,98 & 20,27 & 62,64 \\
\hline E12 & 63 & 72 & 100 & 60 & 80 & 100 & 747,33 & 12,19 & 61,29 \\
\hline E11 & 57 & 58 & 100 & 50 & 44 & 100 & 1514,70 & 39,06 & 38,78 \\
\hline E3 & 71 & 66 & 100 & 81 & 70 & 100 & 557,36 & 21,34 & 26,12 \\
\hline E7 & 61 & 77 & 100 & 75 & 70 & 100 & 580,15 & 40,20 & 14,43 \\
\hline E5 & 70 & 100 & 77 & 82 & 100 & 84 & 325,06 & 32,46 & 10,01 \\
\hline E9 & 100 & 54 & 84 & 100 & 44 & 57 & 1302,81 & 143,68 & 9,07 \\
\hline E8 & 100 & 65 & 88 & 100 & 78 & 99 & 446,85 & 50,43 & 8,86 \\
\hline E1 & 57 & 61 & 100 & 74 & 100 & 97 & 552,20 & 309,13 & 1,79 \\
\hline E10 & 84 & 94 & 100 & 90 & 69 & 100 & 178,27 & 107,87 & 1,65 \\
\hline NE19 & 13 & 13 & 100 & 1 & 15 & 100 & 5380,16 & 21,18 & 253,98 \\
\hline NE24 & 100 & 18 & 37 & 100 & 23 & 28 & 3672,68 & 17,75 & 206,89 \\
\hline NE18 & 52 & 65 & 100 & 43 & 66 & 100 & 1420,45 & 14,05 & 101,10 \\
\hline NE20 & 68 & 67 & 100 & 68 & 75 & 100 & 624,56 & 10,54 & 59,28 \\
\hline
\end{tabular}




\begin{tabular}{c|c|c|c|c|c|c|c|c|c}
\hline NE14 & 100 & 78 & 87 & 100 & 73 & 85 & 298,34 & 5,30 & 56,30 \\
\hline NE17 & 34 & 33 & 100 & 17 & 20 & 100 & 3654,16 & 73,39 & 49,79 \\
\hline NE16 & 100 & 56 & 65 & 100 & 67 & 71 & 848,61 & 25,41 & 33,40 \\
\hline NE23 & 95 & 33 & 100 & 100 & 31 & 75 & 2497,67 & 108,50 & 23,02 \\
\hline NE22 & 82 & 79 & 100 & 66 & 82 & 100 & 361,28 & 44,80 & 8,06 \\
\hline NE13 & 89 & 95 & 100 & 79 & 100 & 96 & 130,33 & 22,12 & 5,89 \\
\hline NE15 & 100 & 25 & 13 & 95 & 100 & 38 & 2581,43 & 1040,92 & 2,48 \\
\hline NE21 & 100 & 93 & 95 & 100 & 70 & 68 & 233,56 & 207,53 & 1,13 \\
\hline
\end{tabular}

Fonte: dados da pesquisa

A Figura 7 ilustra graficamente a decisão do especialista (E2) e do não especialista (NE24), exemplificando tal constatação. Salienta-se que a discussão dos dados, provenientes desses sujeitos experimentais, se justifica pelo fato dos mesmos apresentarem o maior índice CWS entre os participantes do estudo no que se refere decisão em relação à escolha dos apartamentos.
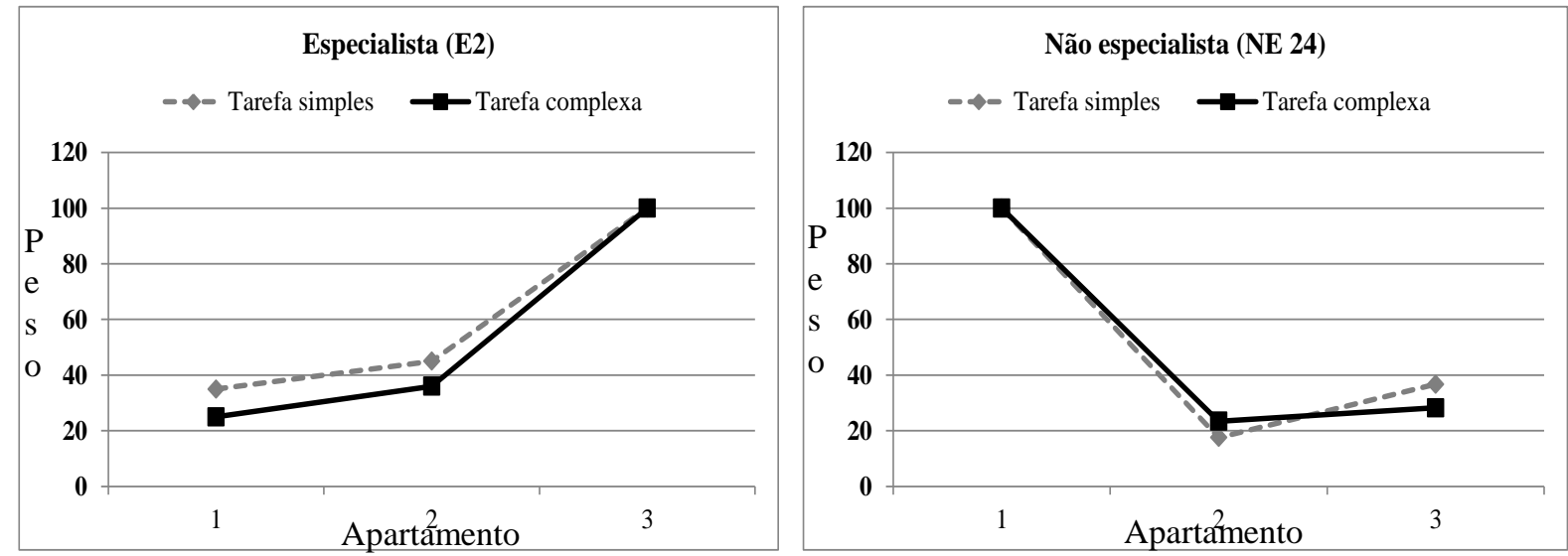

Figura 7 - Resultado da decisão do especialista (E2) e não especialista (NE 24)

Fonte: dados da pesquisa

A Figura 7 demonstra que o não especialista discriminou mais e também apresentou maior consistência na decisão. A discriminação do não especialista foi de 3672,68, enquanto que do especialista foi de 2855,49 . No cálculo da inconsistência, o não especialista apresentou o valor de 17,75 , enquanto para os especialistas o valor foi de 31,62. O índice CWS dos dois indivíduos representados no gráfico (E2 e NE24) foi de 90,32 e 206,89, respectivamente.

Ainda, em relação ao cálculo da média dos índices CWS dos diferentes decisores, o valor médio para discriminação dos não especialistas foi de 1808,60 e inconsistência de 132,62, enquanto a média do CWS para o grupo foi de 45,69. Por outro lado, para os especialistas, o valor médio para discriminação foi de 1045,68 e inconsistência 67,65, sendo a média do CWS 41,27.

A fim de apresentar uma síntese dos resultados obtidos neste estudo, com relação à média dos índices CWS da atribuição de pesos aos critérios e, do resultado da decisão, elaborou-se a Tabela 4. Neste sentido, pode-se observar que, tanto na atribuição de pesos aos critérios, quanto, no resultado da decisão, os não especialistas apresentaram valores superiores do índice CWS quando comparados aos especialistas. Esses achados evidenciaram que os pressupostos defendidos por Shanteau et al. (2003) não foram corroborados na realidade investigada, uma vez que este afirma que indivíduos com maior nível de conhecimento apresentam maior índice CWS, quando comparados a indivíduos novatos (não especialistas).

Tabela 4 - Resumo das médias do CWS por grupos de decisores Média do Índice CWS

\begin{tabular}{l|c|c}
\hline Nível de Conhecimento & Atribuição de peso aos critérios & Resultado da decisão \\
\hline Não Especialista & 12,57 & 45,69 \\
\hline Especialista & 7,99 & 41,27 \\
\hline
\end{tabular}


Com relação à atribuição de pesos aos critérios relevantes na compra de um imóvel, é possível que este tenha sido realizado de acordo com as informações e experiências que os decisores tinham acerca dos mesmos, fazendo com que determinados critérios tivessem maior relevância na sua ponderação. Além disso, os resultados apresentados revelaram importantes particularidades no processo de decisão dos especialistas, os quais demonstraram valores médios de discriminação menores e maiores valores médios de inconsistência, quando comparados aos não especialistas.

Essas constatações podem ser entendidas a partir da Teoria da Imagem, que, segundo Dunegan (1995), é um modelo descritivo do processo de decisão, fundamentado em princípios psicológicos e sociológicos, englobando os grandes paradigmas da decisão comportamental. A Teoria da Imagem postula que o curso de ação na escolha do decisor dá-se em função da percepção de várias imagens que ele possui, incluindo a imagem de valor, de trajetória e estratégica. A imagem de valor refere-se aos princípios e crenças do decisor; a imagem da trajetória diz respeito aos objetivos e metas futuras do decisor e a imagem estratégica consiste em planos e táticas do decisor para atingir seus objetivos. Dessa forma, considerando a Teoria da Imagem, o decisor possui uma imagem de uma boa solução na sua mente e, no processo de decisão, procura uma solução que atinja o objetivo previamente estabelecido (LÖBLER, 2005).

\section{Considerações finais}

A fim de avaliar a consistência e a discriminação de indivíduos com diferentes níveis de conhecimento no processo decisório, considerando a complexidade da tarefa, realizou-se um estudo, por meio de um experimento, cuja tarefa decisória consistia na simulação de compra de um imóvel residencial (apartamento). Dessa forma, os indivíduos, auxiliados por um Sistema de Apoio à Decisão (DECISOR) e por um material ilustrativo (folders), atribuíram pesos aos oito critérios (preço, localização, área privativa, padrão de acabamento, posição solar, posição em relação ao andar, posição do apartamento no prédio e número de banheiros) considerados relevantes no processo de escolha de um imóvel.

A apresentação dos resultados exibiu diferenças entre os níveis de conhecimento dos decisores. Nesse sentido, por meio do cálculo do índice CWS (Cochran, Weiss e Shanteau), verificouse que, de maneira geral, o CWS dos indivíduos não especialistas foi maior do que o CWS dos especialistas, ou seja, os não especialistas discriminaram mais e foram menos inconsistentes na atribuição de peso aos critérios, ao analisarem as alternativas da tarefa simples e posteriormente da tarefa complexa. Por outro lado, os indivíduos especialistas discriminaram menos e foram mais inconsistentes em relação aos não especialistas.

No que se refere ao resultado da decisão, os indivíduos não especialistas mantiveram o mesmo parâmetro da atribuição de pesos aos critérios, uma vez que discriminaram mais e foram mais consistentes, resultando em um índice CWS maior do que os especialistas. Com relação à média dos índices CWS dos diferentes decisores, percebeu-se que tanto na atribuição de peso aos critérios, quanto no resultado da decisão, os não especialistas discriminaram mais e foram mais consistentes.

Diante desses resultados, é possível deduzir que a ponderação dos critérios foi motivada pelo que os decisores julgavam mais relevante no momento da sua escolha. Neste escopo, a discussão dos resultados apresentados não permitiu confirmar os pressupostos defendidos por Shanteau et al. (2003) no que tange à discriminação e inconsistências de especialistas e novatos.

Essas constatações podem estar associadas à Teoria da Imagem, a qual, segundo Dunegan (1995), o curso de ação na escolha do decisor dá-se em função da percepção de várias imagens que possui. Assim, neste estudo, a imagem de valor dos indivíduos pode estar associada a suas crenças em relação a aspectos mercadológicos, como, por exemplo, localização privilegiada na cidade onde atuam. Considerando a imagem estratégica, outro aspecto que pode ter influenciado a decisão dos indivíduos está relacionado à sua experiência, o que fez com que os mesmos atribuíssem peso aos critérios, visando à alternativa que já haviam definido em sua mente.

Ainda, pode-se deduzir que a maneira com que os especialistas analisaram a tarefa e atribuíram pesos aos critérios pode ter sido em detrimento de vieses cognitivos, utilizados no processo de escolha, visto que, os indivíduos talvez não tenham conseguido dissociar o processo de venda de imóveis quando realizado a clientes, em detrimento do processo de compra de um imóvel para 
residência própria. Neste sentido, segundo Kahnemann (1991) existem evidências de que indivíduos especialistas não estão imunes às ilusões cognitivas que, segundo ele, são universais.

Adicionalmente ao que foi discutido, é importante mencionar as limitações deste estudo. Talvez a principal limitação seja em decorrência do tipo de pesquisa aplicada, que foi a experimental, tendo em vista a dificuldade de reproduzir situações da vida real, representada, neste estudo, pela simulação da compra de um apartamento, mantendo o total controle das variáveis. Destaca-se também a possibilidade de viés, provocada por efeito de teste, pelo fato de os sujeitos experimentais serem submetidos a diferentes condições em uma sequência particular. Ainda, há uma limitação relacionada diretamente ao índice CWS, pois os próprios autores do método sugerem que a utilização de outras formas de mensuração pode contribuir com a eficácia do CWS.

Considerando tais resultados, sugere-se a replicação do estudo com outro tipo de tarefa, bem como em um contexto mais realista, não em uma situação hipotética. Ainda, a realização de pesquisas futuras poderia enfocar a utilização de outro sistema de apoio à decisão, bem como o uso do protocolo verbal think aloud, visando ampliar o entendimento a cerca das estratégias utilizadas no processo decisório dos indivíduos.

\section{Referências}

AAKER, D. A.; KUMAR, V.; DAY, G. S. Pesquisa de Marketing. 2. ed. São Paulo: Atlas, 2004, $745 \mathrm{p}$.

BEDARD, J.; CHI, M.T.H.; GRAHAM, L.E.; SHANTEAU, J. Expertise in auditing: discussing. Auditing, v.12, n.21, p. 21-45, 1993.

CHASE, W.G.; SIMON, H. A. Perception in chess. Cognitive Psychology, v.4, pp.55-81, 1973.

CHEN, S. Y.; FAN, J. P.; MACREDIE, R.D. Navigation in hypermedia learning systems: experts vs. novices. Computers in Human Behavior, v.22, n.1, p. 251-266, 2006.

COZBY, P. C. Métodos de pesquisa em ciências do comportamento. São Paulo: Atlas, 2003, 454p.

COCHRAN, W. G. The comparison of different scales of measurement for experimental results. Annals of Mathematical Statistics, v.14, pp.205-216, 1943.

DUNEGAN, K. J. Image Theory: Testing the Role of Image Compability in Progress Decisions. Organizational Behavior and Human Decision Processes. v. 62, n.1, p. 354- 375, 1995.

EINHORN, H. J. Expert Judgment: some necessary conditions and an example. Journal of applied psychology, v.59, 1974.

ERICSSON, A. K. The Influence of Experience and Deliberate Practice on the Development of Superior Expert Performance. In: ERICSSON, K. A.; CHARNESS, N., FELTOVICH, P.; HOFFMAN, R.R. (Eds.). Cambridge handbook of expertise and expert performance. Cambridge, UK: Cambridge University Press, 2006. p.685- 705.

FISHER, C.W., CHENGALUR-SMITH, I.; BALLOU, D.P. The impact of experience and time on the use of data quality information in decision making. Information Systems Research, v.14, n.2, p.170$189,2003$.

GERMAIN, M.L. A chronological synopsis of the dimensions of expertise: toward the expert of the future. Performance Improvement, v. 50, n. 7, p. 38-46, 2011.

IMOFAR, Imobiliária. Folder ilustrativo da alternativa de apartamento Hamburgo. Disponível em: <http://www.imofar.com.br/detalhes.php?codigo=3870>; Acesso em: 09 janeiro de 2013. 
JENKINS, C.; CORRITORE, C. L.; WIEDENBECK, S. Patterns of information seeking on the web: A qualitative study of domain expertise and Web expertise. Information Technology and Society, v.1, n.3, p.64-89, 2003.

KAHNEMAN, D. Judgment and decision making: a personal view. Psychological Science. v. 2, n. 3, p.142-145, 1991.

LÖBLER, M. L. Processamento da Informação: Uma Avaliação dos Diferentes Níveis de Conhecimento no Processo de Decisão. 2005. 215f. Tese (Doutorado em Administração) Universidade Federal do Rio Grande do Sul, Porto Alegre, 2005.

LÖBLER, M.L.; HOPPEN,N. Discriminação e consistência no processo decisório: mensuração do nível de conhecimento utilizando o índice CWS. Produto \& Produção, v. 9, n.3, p. 95-108, 2008.

MALHOTRA, V. A.; LEE, M. D.; KHURANA, A. Domain experts influence decision quality: Towards a robust method for their identification. Journal of Petroleum Science and Engineering, v.57, n.2, p.181-194, 2007.

MARKMAN, A. B.; MEDIN, D. L. Decision Making. In: MEDIN, D.L., ROSS, B.H.; MARKMAN, A.B. Cognitive Psychology . Fort Worth, TX: Harcourt Brace, 2001. p.413-466.

OLIVEIRA, L. S. M.; CORREIA, T. C. V. D.; de MELLO, J. C. C. B. S. Métodos Multicritério de Auxílio à Decisão aplicados a avaliação e aquisição de imóveis. Relatórios de Pesquisa em Engenharia de Produção, v. 8 n.4, 2008.

PATEL, V. L.; GROEN, G. L. The generality of medical expertise: a critical look. In: ERICSON, A; SMITH, J. Toward a General Theory of Expertise: Prospects and Limits, New York, NY: Cambridge University Press, 1991. p.93-126.

PAULEY, K.; OHARE, D.; WIGGIND, M. Measuring Expertise in Weather-Related Aeronautical Risk Perception: The Validity of the Cochran- Weiss Shanteau (CWS) Index. The International Journal of Aviation Psychology, v.19, n.3, p. 201-216, 2009.

SHANTEAU, J.; WEISS, D.; THOMAS, R.; POUNDS, J. How Can You Tell if Someone is an Expert? Empirical Assessment of Expertise. In: SCHNEIDER, S.L.; SHANTEAU, J. Press). Emerging perspectives on judgment and decision research. Cambridge, Cambridge University Press, 2003. p. 39-62.

SINDICATO DAS EMPRESAS DE COMPRA, VENDA, LOCAÇÃO E ADMINISTRAÇÃO DE IMÓVEIS COMERCIAIS E RESIDENCIAIS DE SÃO PAULO. Boas práticas na intermediação de compra e venda de imóveis. São Paulo: Autores. Disponível em: < http://www.secovi.com.br/files/Downloads/manual-boas-praticas-webpdf.pdf>. Acesso em: 12 fevereiro de 2013.

SIMMONS, P. E., LUNETTA, V. N.. Problem-solving behaviors during a genetics computer simulation: Beyond the expert/novice dichotomy. Journal of Research in Science Teaching, v.30, n.2, p. 153-173, 1993.

SPIRES, H. A. ; DONLEY, J. Prior knowledge activation: Inducing engagement with informational texts. Journal of Educational Psychology, v.90, n.2, p. 249-260, 1998.

STERNBERG, R. J. Psicologia cognitiva. Porto Alegre: Artes médicas, 2000. 352p.

WEISS, D.J.; SHANTEAU, J.; HARRIES, P. People Who Judge People. Journal of Behavioral Decision Making, v. 19, n. 2, p. 441-454, 2006. 
WEISS, D. J.; SHANTEAU, J. CWS: A User's Guide. Disponível em: < http://www.researchgate.net/profile/James_Shanteau/publication/262915204_CWS_A_User's_Guide/l inks/0046353937c73193c7000000.pdf>; Acesso em: 08 janeiro de 2013.

WITTEMAN, C.L.M. WEISS, D.J.; METZMACHER, M. Assessing Diagnostic Expertise of Counselors Using the Cochran-Weiss-Shanteau (CWS) Index. Journal of Counseling \& Development, v.90, n.1, p.30-38, 2012. 